\title{
Which Factors Are Associated with Positive Resection Margin in Pancreatic Ductal Adenocarcinoma for different surgical procedures? Results Based on a Standard Pathological Evaluation System-Retrospective Cohort Study
}

Bo Li

Changhai Hospital

Shiwei Guo

Changhai Hospital

Xiaohan Shi

Changhai Hospital

Chenming Ni

Changhai Hospital

Suizhi Gao

Changhai Hospital

Gang Li

Changhai Hospital

\section{Canrong Ni}

Changhai Hospital

Hui Jiang

Changhai Hospital

Wan Yee Lau

Prince of Wales Hospital

Gang Jin ( $\nabla_{\text {jingang@smmu.edu.cn ) }}$

Changhai Hospital https://orcid.org/0000-0001-6713-1185

\section{Research Article}

Keywords: Distal pancreatosplenectomy, Pancreatic cancer, Pancreaticoduodenectomy, Risk factor, Resection margin

Posted Date: May 5th, 2021

DOI: https://doi.org/10.21203/rs.3.rs-111309/v2

License: (c) (i) This work is licensed under a Creative Commons Attribution 4.0 International License. Read Full License 


\section{Abstract}

Background/Objectives: The present study identified the independent risk factors of R1 resection in pancreaticoduodenectomy (PD) and distal pancreatosplenectomy (DP) for patients with pancreatic ductal adenocarcinoma (PDAC).

Methods凹Consecutive patients who were operated from $1^{\text {st }}$ December 2017 to $30^{\text {th }}$ December 2018 with curative intent were analyzed retrospectively. A standardized pathological examination with digital whole-mount slide images (DWMSIs) was utilized for the resection margin status. R1 was defined as microscopic tumor infiltration within $1 \mathrm{~mm}$ to the resection margin. The potential risk factors of R1 resection for PD and DP were analyzed separately by univariate and multivariate logistic regression analyses.

Results囚For the 240 patients who underwent PD, and the 146 patients who underwent DP, the R1 resection rates were $30.8 \%$ and $35.6 \%$, respectively. Univariate analysis on risk factors of R1 resection for PD were tumor location, absence of tumor necrosis, N staging, and TNM staging; while those for DP were perineural invasion, T staging, and TNM staging. Multivariate logistic regression analysis showed the location of tumor in the neck and uncinate process, and N1/2 staging were independent risk factors of R1 resection for PD; while those for DP were T3/4 staging.

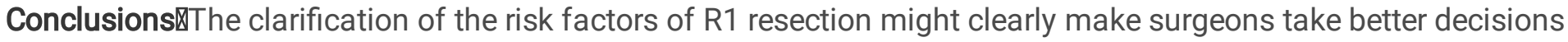
on surgical strategies for different surgical precedures in patients with PDAC.

\section{Introduction}

Pancreatic cancer is a lethal disease, being characterized by increasing incidences and mortality rates, and with a dismayed 5-year survival rate of $<9 \%[1,2]$. Currently, radical surgery is the only potentially curative therapy for pancreatic ductal adenocarcinoma (PDAC) [3]. However, only about 15-20\% of patients are eligible for upfront radical resection at the time of diagnosis $[4,5]$. Even when surgery is followed by chemotherapy or radiotherapy, the majority of patients develop tumor recurrence within 2 years after surgery [6]. Tumor relapse can occur either locally or as a metastatic disease [7]. Recurrence relating to microscopic positive resection margins (R1) is common, accounting for $46.7 \%$ of patients with pancreatic body/tail and $8.2 \%$ with pancreatic head PDAC [8]. Recent studies showed that the R1 margin status significantly lowered the disease-free survival (DFS) and the overall survival (OS) compared to the clear resection (R0) margin status after pancreaticoduodenectomy (PD) [9] or distal pancreatosplenectomy (DP) [10]. Thus, to achieve R0 resection is critical to prognosis of patients. The risk factors of $\mathrm{R} 1$ resection should be identified to improve patients' management.

The independent risk factors of R1 resection in PD and DP for PDAC have not been extensively investigated. Furthermore, the definition of R1 and the protocol on pathological examination of resected specimens have not been standardized [11-13]. In our high volume pancreatic center, R1 is defined as detection of any cancer cells within $1 \mathrm{~mm}$ of one or more surfaces or margins in the resected specimens. Meanwhile, the Leeds Pathology Protocol (LEEPP) was used to standardize our protocol of pathological examination since November 2016. The pathological reports were rigorously filed following the latest National Comprehensive Cancer Network (NCCN) guidelines (Version 1, 2021) for pancreatic adenocarcinoma. The R status has since been assessed by digital whole-mount slide images (DWMSIs) which has the advantages of identifying the R status more precisely and comprehensively compared to the ordinary microscopic study. The present study, based on a standardized protocol of pathological 
examination and R1 definition, aimed to identify independent risk factors of R1 resection for PD and DP in patients with PDAC. These findings, hopefully, could provide useful information for clinical practice.

\section{Methods}

\section{Study population}

This retrospective study included consecutive patients who were diagnosed to have PDAC by pathological examination after pancreatic resectional surgery between $1^{\text {st }}$ December 2017 to $30^{\text {th }}$ December 2018 . The inclusion criteria were: (1) PD or DP with curative intention, and (2) underwent a standardize pathological protocol for the resected specimen and a standard reporting record for resection margin (R) status according to the NCCN guidelines for pancreatic cancer. The exclusion criteria were: (1) total pancreatectomy (TP); (2) macroscopic evidence of margin involvement (R2); (3) patients received neoadjuvant chemotherapy; (4) resection for metastatic disease. The Changhai Hospital Review Board approved the study, and no additional informed consent was required to review the patients' medical records.

\section{Pathological Examination}

The Leeds Pathology Protocol (LEEPP) was routinely used $[14,15]$ for the pathological examination.

\section{For PD specimens:}

Briefly, after multicolor inking of the posterior, medial margin, and anterior surfaces of the pancreatic head, the specimen of PD was serially sliced in an axial plane, perpendicular or paralleled to the longitudinal duodenal axis.

\section{For DP specimens:}

After multicolor inking of the posterior and anterior surfaces of the pancreatic body and tail, the specimen of DP was serially sliced in a plane parallel to the main pancreatic duct or the pancreatic transection margin when the tumor invades to the surface of the pancreas according to the NCCN guidelines.

The entire specimen was sliced in 5-mm-thick sections, which resulted in 10-35 (average 24.5 \pm 6.7 ) formalin-fixed paraffin-embedded (FFPE) block for each specimen. Then, each FFPE block was cut in 4-mm-thick sections on whole-tissue glass slides measuring $7.8 \times 5.4 \mathrm{~cm}^{2}$. These slides were scanned by the Hamamatsu S60 Whole Slide Scanner (Hamamatsu Photonics, Hamamatsu city, Japan) to obtain the DWMSIs with an average of $6.47 \mathrm{~GB}$ in file size [16]. The DWMSIs can also be observed by the NanoZoomer Digital Pathology (NDP) viewer2 version 2.7.25 (Figure 1), and the distance between the resection margins and the tumor cells can be measured precisely down to $0.01 \mathrm{~mm}$ on the screen (Supplementary Figure 1).

\section{Classification and Defining of the R Status}

The pathological reports on a detailed form were reassessed retrospectively. The slides were rechecked if the reports were not clearly depicted. According to the NCCN guidelines, the R status records for PD were comprised of the transection margin, the anterior surface, the posterior margin, the bile duct margin, the enteric margin, and the medial margin, which included the SMV (superior mesenteric vein)/PV (portal vein) resection margin and the SMA (superior mesenteric artery) resection margin. There were just three margins for DP. R1 was defined as one or more 
cancer cells within $1 \mathrm{~mm}$ of one or more surfaces or margins (R1<1 mm) or at the surface or margin (R1-directed). $\mathrm{RO}$ was defined as $>1 \mathrm{~mm}$ from any surface or margin $(\mathrm{RO}>1 \mathrm{~mm})$.

\section{Variables Analyzed}

For all the patients, the following demographic, clinical, and pathological variables, including sex, age, preoperative carbohydrate antigen (CA) 19-9, tumor location (head, neck, or uncinate process for PD; neck and body, or body and tail for DP), necrosis, perineural invasion, lymphovascular invasion, duodenal papilla invasion, tumor grade (G1/2 or G3/4), vascular resection. The TNM staging was also recorded using the $8^{\text {th }}$ edition of the AJCC Cancer Staging Manual for Pancreatic Cancer. Resectability status was recorded according to the NCCN guideline for pancreatic adenocarcinoma (version 3. 2021).

\section{Statistical Analysis}

Categorical data were presented as percentage proportion, and continuous data were transformed into categorical data by the median value. For incomplete data, a dummy variable classified as "missing" was included in the analysis. To compare the differences in the categorical data between the two groups, the Fisher's exact test or the $c^{2}$ test was used as appropriate. All variables found to be statistically significant $(P<0.1)$ on univariate analysis were subsequently included in a multivariate binary logistic regression model to determine the independent risk factors associated with $\mathrm{R} 1$ resection. For all the analyses, a two-tailed $P<0.05$ was considered as statistically significant. The analyses were performed using the SPSS version 25.0 (SPSS, Chicago, IL, USA).

\section{Results}

\section{Patient Characteristics}

From $1^{\text {st }}$ December 2017 to $30^{\text {th }}$ December 2018, 457 patients underwent pancreatic surgery and were diagnosed to have PDAC on pathological examination. A total of 71 (15.5\%) patients were excluded: 6 patients because of TP, 7 patients due to a lack of standard records for the R status, 9 patients because of R2, 41 patients because of neoadjuvant chemotherapy before surgery, and 8 patients because of resection for metastatic disease, which were discovered during the operations. The remaining 386 patients were included in this study ( $M: F=1.47: 1$; age range 2883 , mean age $61.9 \pm 9.5$ years), with 240 patients underwent PD and 146 patients underwent DP (Figure 2). The R status of the two groups of patients was assessed using DWMSIs. No significant differences were detected in the baseline data between R0 and R1 resections in the patients who underwent PD and DP, respectively.

\section{Distribution of the $R$ Status (margins)}

For all the patients, 260 (67.4\%) patients had a R0 margin, 77 (19.9\%) patients a R1<1 mm margin, and 49 (12.7\%) patients a R1-directed margin (Table 1). Table 1 also showed that for the 240 patients who underwent PD, 74 $(30.8 \%)$ patients had R1 resections (either R1 $<1 \mathrm{~mm}$ or R1-directed). A single positive margin was found in 52 (21.7\%) patients, two positive margins in 14 (5.8\%) and $\geq 3$ positive margins in 8 (3.3\%). The R1 resection incidence rates of the six specific margins in PD were $19.6 \%$ in the medial margin, $10.5 \%$ in the anterior surface, $9.6 \%$ in the posterior margin, $3.8 \%$ in the transection margin of pancreas, $0.8 \%$ in the bile duct margin, and none in the enteric margin. The R1 resection in the medial margin of the PD was maximal (44.3\%) of the total R1 resection margins, followed by the anterior surface (23.6\%) and the posterior margin (21.7\%). For the 146 patients who underwent DP, 52 had R1 resections (35.6\%). A single positive margin was found in 35 patients (24.0\%) and two positive margins 
in 17 patients (11.6\%). The R1 resection incidence rates of the anterior surface was $26.0 \%$, the posterior surface $19.2 \%$, and the transection margin of pancreas $2.1 \%$. In DP, the R1 resection of the anterior surface was maximal $(55.1 \%)$, followed by the posterior margin (40.6\%) (Table 1$)$.

Univariate Analysis of Risk Factor for R1 Resection

Univariate analysis on demographics and potential risk factors between the R0 and R1 resection groups of patients who underwent PD are shown in Table 2. Patients with R1 resection had a higher incidence of tumor localization in the neck and uncinate process $(40.5 \%$ vs. $16.3 \%, \mathrm{P}<0.001)$ and lymphovascular invasion ( $50 \%$ vs. $36.7 \%, \mathrm{P}=0.054)$. Duodenal papilla invasion were common in patients with $\mathrm{R} 0$ and $\mathrm{R} 1$ resections $(28.3 \%$ vs. $17.6 \%, \mathrm{P}=0.076)$. N1/2 cancer (83.8\% vs. 57.2\%, $\mathrm{P}<0.001)$ and TNM III cancer (48.6\% vs. 21.7\%, $\mathrm{P}<0.001)$ were significantly higher in the R1 resection group as compared to the $\mathrm{RO}$ group. Univariate analysis on demographics and potential risk factors between the R0 and R1 resection groups of patients who underwent DP are also shown in Table 2. Patients with R1 resection had a high incidence of perineural invasion $(96.2 \%$ vs. $85.1 \%, P=0.041)$. The prevalence of T3 cancer (76.9\% vs. 42.6\%, $\mathrm{P}<0.001)$ and TNM stage III cancer (34.6\% vs. 19.1\%, $\mathrm{P}=0.038)$ were significantly higher in the $\mathrm{R} 1$ resection group as compared to the R0 group. The prevalence of $\mathrm{N} 1 / 2$ cancer $(69.2 \%$ vs. $56.4 \%, \mathrm{P}=0.095)$ was higher in the $\mathrm{R} 1$ resection group.

\section{Multivariable Analysis of Risk Factor for R1 Resection}

Multivariable analyses showed that neck tumors (odds ratio $(O R)=9.549, P=0.001$ ) uncinate process tumor $(\mathrm{OR}=3.311, \mathrm{P}=0.002)$, and $\mathrm{N} 1(\mathrm{OR}=2.406, \mathrm{P}=0.031)$ and $\mathrm{N} 2(\mathrm{OR}=6.430, \mathrm{P}<0.001)$ staging were independent risk factors of R1 resection in patients who underwent PD. Multivariable analyses showed that T3 staging $(O R=5.26$, $\mathrm{P}<0.001$ ) was an independent risk factor of $\mathrm{R} 1$ resection in patients who underwent DP (Table 3).

\section{Discussion}

Previous reports have shown a close relationship between R1 resection and OS (or DFS) in PDAC patients [10, 1719]. Even for patients who underwent PD for PDAC with positive resection margins shown on frozen section, further surgical resection to achieve RO did not have any significant positive impact on OS [20]. These studies suggested that it is vital to achieve $\mathrm{RO}$ resection on the first attempt of surgical resection. Knowledge on independent risk factors of R1 resection can help surgeons to make better decisions on resection strategies.

Studies on independent risk factors of R1 resection was done difficultly because of the differences in the definition between the Union for International Cancer Control (UICC) criteria (R1: $0 \mathrm{~mm}$ definition) and the UK Royal College of Pathologists (RCPath) criteria (R1: $1 \mathrm{~mm}$ definition) [21]. Furthermore, most studies on risk factors of R1 resection did not apply a standard pathological examination protocol, thus making any comparisons among these studies impossible. Also, most studies focused on pancreatectomy and did not discriminate between PD and DP. The risk factors of R1 resection in these two different surgical operations for PDAC differ significantly.

With accurate assessment of resection margins by a standardized pathological examination protocol using LEEPP and DWMSIs, and with a single definition of R1 using the $1 \mathrm{~mm}$ definition, our study showed marked differences in the $\mathrm{R} 1$ resection rates for PD and DP when compared with the published rates of R1 resection [22-26]. In the current study, the rates of R1 resection were similar to the recently reported data for patients who underwent upfront surgery [27]. Previous studies reported R1 resection was frequently present in PV/SMV margins [28] and SMA margins[29], which was closely related to prognosis [30]. Our data also showed similar results in PD. Previous studies who 
demonstrated lesions in the neck $(\mathrm{OR}=5.48)$ or uncinate process $(\mathrm{OR}=2.996)$ [11], tumor size $>30 \mathrm{~mm}(\mathrm{OR}=1.13)$ [12] and grade 3 tumors $(\mathrm{OR}=4.05)$ [28] were independent risk factors of $\mathrm{R} 1$ resection. However, there were no studies to clarify independent risk factors separately for R1 in PD and DP. The NCCN guidelines for pancreatic adenocarcinoma recommend operation and resection margins should be assessed separately in PD and DP. Our study supported neck/uncinate tumor, and the N1/2 stage were independent risk factors of R1 resection for PD, and T3 were independent risk factors for DP.

The pancreatic neck is a narrow anatomical region between the pancreatic head and body, lying just anterior to the PV, gastroduodenal artery, and common hepatic artery [31]. The uncinate process is closely related to the SMA and the SMV [32]. As a consequence, pancreatic neck or uncinate process cancer are prone to invade these major vessels to become either a borderline or unresectable tumor. R0 resection is technically difficult with involvement of theses vessels. In our study, neck tumor had the highest R1 resection rate, followed by uncinate process tumors. In addition, the most frequently R1 invaded margin in PD was the medial margin which included the SMA margin and SMV/PV margin. When the R status of different tumor locations in PD was analyzed (Supplementary Table 1), neck tumors had a significantly higher rate of positive resection margin in the transection margins (38.5\%) and medial margins (53.8\%). These results suggested that the R0 resection rate of neck tumor was extremely low (3/13 or $23.1 \%)$. Our results suggested that surgeons should resect more pancreatic tissues towards the pancreatic tail or even to do TP. To decrease R1 resection in the medial margin, the alternative treatment for patients with pancreatic neck tumor should undergo neoadjuvant therapy followed by surgery. Supplementary Table 1 also showed that uncinate process tumors had a high frequency (31.8\%) of positive medial margins. Besides, it may explain that the pancreatic tumor cells are characterized by neurotropic features[33], and the tumor located in uncinate process are more likely invaded the neural structures. The regional lymph nodes are mainly distributed along vasculature, and tumor cells can easily invade lymph nodes along the lymphatic drainage pathway. This explain why the $\mathrm{N}$ stage is an independent risk factor of R1 resection in PD. In further analysis showed that tumors with N2 stage had a higher frequency $(32.9 \%)$ of positive medial margins compared with N0 (10.8\%) or N1 (17.2\%), so as posterior margin with $22.9 \%$ of R1 in N2 stage (Supplementary Table 1), which were also matched with pathway of lymph nodes metastasis.

Pancreatic body and tail tumors present late as they do not have symptoms in the early stages [34]. Thus, the pancreatic body and tail tumors are likely to invade beyond the pancreas. Our study suggested that T3 was an independent risk factor of R1 resection. And the anterior surface is more vulnerablely invaded by the tumor with R1 resection rate of $36.3 \%$ in T3 stage (Supplementary Table 2), which may indicate that the surgical procedure related with anterior surface of pancreas should exclusively fit the principles of an-neoplasia surgical operation and antouch radical excision, in order to avoid the implantation metastasis during the operation.

The present study has several limitations. First, this retrospective study has the intrinsic defects of any retrospective study. Second, the definition of R1 resection margin and the standard procedure for pathological examination of resection margin used in this study made it difficult to compare our results with other published studies. Third, longterm surgical outcomes could not be obtained due to the short study period. Fourth, we used surgical knife to cut the pancratic tissye in the PD, whereas the staple was applied to separate the tumor, and the different processing of pancreatic transection margin may influenced $\mathrm{R}$ status evaluation.

\section{Conclusions}


A standardized pathological examination using the LEEPP, DWMSIs and the definition of R1 resection margin within $1 \mathrm{~mm}$ to the tumor were used in this study to analyze independent risk factors for PD and DP separately. The clarification of the risk factors of $\mathrm{R} 1$ resection might clearly make surgeons take better decisions on surgical strategies for different surgical precedures of pancreatic resection.

\section{Declarations}

\section{Ackowledgments}

We would like to thank Prof. Wan Yee Lau for English language polishing.

\section{Authors' contributions}

LB,GSW and SXH contributed to analysis and interpretation of data, drafting the article and final approval of the version to be published. NCM, GSZ, LG and NCR contributed to conception and design of data, revising the article critically for important intellectual content and final approval of the version to be published. JH, LWY and JG contributed to analysis and interpretation of data, drafting the article and final approval of the version to be published. All authors read and approved the final manuscript.

\section{Funding}

This work was supported by the National Natural Science Foundation of China (grant number 81972913), the Top Project of the Military Medical Science and Technology Youth Training Program (grant number 16QNP095) and Clinical Research Plan of SHCD (grant number SHDC2020CR2001A).

\section{Availability of data and materials}

Please contact the corresponding authors for data requests.

\section{Ethics approval and consent to participate}

The study was approved by the Institutional Review Board of Changhai Hospital (Shanghai Changhai Hospital Ethics Committee, reference number: CHEC2020-043).

\section{Consent for publication}

All patients enrolled in the study signed the consent for publication. The written consent is available for review by the Editor-in-Chief of this journal.

\section{Competing interests}

The authors declare that they have no competing interests.

\section{References}

1. Chhoda A, Lu L, Clerkin BM, Risch H, Farrell JJ: Current Approaches to Pancreatic Cancer Screening. The American journal of pathology 2019, 189(1):22-35.

2. Siegel RL, Miller KD, Jemal A: Cancer statistics, 2019. CA: A Cancer Journal for Clinicians 2019, 69(1):7-34. 
3. Hackert T, Ulrich A, Buchler MW: Borderline resectable pancreatic cancer. Cancer letters 2016, 375(2):231-237.

4. Speer AG, Thursfield VJ, Torn-Broers Y, Jefford M: Pancreatic cancer: surgical management and outcomes after 6 years of follow-up. The Medical journal of Australia 2012, 196(8):511-515.

5. Conroy T, Bachet JB, Ayav A, Huguet F, Lambert A, Caramella C, Marechal R, Van Laethem JL, Ducreux M: Current standards and new innovative approaches for treatment of pancreatic cancer. European journal of cancer (Oxford, England : 1990) 2016, 57:10-22.

6. Tummers WS, Groen JV, Sibinga Mulder BG, Farina-Sarasqueta A, Morreau J, Putter H, van de Velde CJ, Vahrmeijer AL, Bonsing BA, Mieog JS et al: Impact of resection margin status on recurrence and survival in pancreatic cancer surgery. The British journal of surgery 2019, 106(8):1055-1065.

7. Liu X, Fu Y, Chen Q, Wu J, Gao W, Jiang K, Miao Y, Wei J: Predictors of distant metastasis on exploration in patients with potentially resectable pancreatic cancer. BMC Gastroentero/ 2018, 18(1):168.

8. Kovac JD, Mayer P, Hackert T, Klauss M: The Time to and Type of Pancreatic Cancer Recurrence after Surgical Resection: Is Prediction Possible? Academic radiology 2019, 26(6):775-781.

9. Adamu M, Nitschke P, Petrov P, Rentsch A, Distler M, Reissfelder C, Welsch T, Saeger HD, Weitz J, Rahbari NN: Validation of prognostic risk scores for patients undergoing resection for pancreatic cancer. Pancreatology : official journal of the International Association of Pancreatology (IAP) [et al] (2018), S1424-3903(18)30110-8.

10. Hank T, Hinz U, Tarantino I, Kaiser J, Niesen W, Bergmann F, Hackert T, Buchler MW, Strobel O: Validation of at least $1 \mathrm{~mm}$ as cut-off for resection margins for pancreatic adenocarcinoma of the body and tail. The British journal of surgery 2018, 105(9):1171-1181.

11. Lai CC, Wang SY, Liao CH, Hsu JT, Chiang KC, Yeh TS, Hwang TL, Yeh CN: Surgical Margin Status of Patients with Pancreatic Ductal Adenocarcinoma Undergoing Surgery with Radical Intent: Risk Factors for the Survival Impact of Positive Margins. In vivo (Athens, Greece) 2018, 32(6):1591-1597.

12. Cassinotto C, Dohan A, Zogopoulos G, Chiche L, Laurent C, Sa-Cunha A, Cuggia A, Reinhold C, Gallix B: Pancreatic adenocarcinoma: A simple CT score for predicting margin-positive resection in patients with resectable disease. European journal of radiology 2017, 95:33-38.

13. Hong SB, Lee SS, Kim JH, Kim HJ, Byun JH, Hong SM, Song KB, Kim SC: Pancreatic Cancer CT: Prediction of Resectability according to NCCN Criteria. Radiology 2018, 289(3):710-718.

14. Verbeke CS, Leitch D, Menon KV, McMahon MJ, Guillou PJ, Anthoney A: Redefining the R1 resection in pancreatic cancer. The British journal of surgery 2006, 93(10):1232-1237.

15. Verbeke CS: Resection margins and R1 rates in pancreatic cancer-are we there yet? Histopathology 2008, 52(7):787-796.

16. Farris $A B$, Cohen $C$, Rogers $T E$, Smith GH: Whole Slide Imaging for Analytical Anatomic Pathology and Telepathology: Practical Applications Today, Promises, and Perils. Archives of pathology \& laboratory medicine 2017, 141(4):542-550.

17. Osipov A, Nissen N, Rutgers J, Dhall D, Naziri J, Chopra S, Li Q, Hendifar AE, Tuli R: Redefining the Positive Margin in Pancreatic Cancer: Impact on Patterns of Failure, Long-Term Survival and Adjuvant Therapy. Ann Surg Oncol 2017, 24(12):3674-3682.

18. Ghaneh P, Kleeff J, Halloran CM, Raraty M, Jackson R, Melling J, Jones O, Palmer DH, Cox TF, Smith CJ et al: The Impact of Positive Resection Margins on Survival and Recurrence Following Resection and Adjuvant Chemotherapy for Pancreatic Ductal Adenocarcinoma. Annals of surgery 2019, 269(3):520-529. 
19. de Geus SWL, Kasumova GG, Sachs TE, Ng SC, Kent TS, Moser AJ, Vahrmeijer AL, Callery MP, Tseng JF: Neoadjuvant therapy affects margins and margins affect all: perioperative and survival outcomes in resected pancreatic adenocarcinoma. HPB (Oxford) 2018, 20(6):573-581.

20. Dikmen K, Kerem M, Bostanci H, Sare M, Ekinci O: Intra-Operative Frozen Section Histology of the Pancreatic Resection Margins and Clinical Outcome of Patients with Adenocarcinoma of the Head of the Pancreas Undergoing Pancreaticoduodenectomy. Med Sci Monit 2018, 24:4905-4913.

21. Markov P, Satoi S, Kon M: Redefining the R1 resection in patients with pancreatic ductal adenocarcinoma. Journal of hepato-biliary-pancreatic sciences 2016, 23(9):523-532.

22. Yamamoto $\mathrm{T}$, Uchida $\mathrm{Y}$, Terajima $\mathrm{H}$ : Clinical impact of margin status on survival and recurrence pattern after curative-intent surgery for pancreatic cancer. Asian journal of surgery 2019, 42(1):93-99.

23. Rau BM, Moritz K, Schuschan S, Alsfasser G, Prall F, Klar E: R1 resection in pancreatic cancer has significant impact on long-term outcome in standardized pathology modified for routine use. Surgery 2012, 152(3 Suppl 1):S103-111.

24. Nitschke P, Volk A, Welsch T, Hackl J, Reissfelder C, Rahbari M, Distler M, Saeger HD, Weitz J, Rahbari NN: Impact of Intraoperative Re-resection to Achieve R0 Status on Survival in Patients With Pancreatic Cancer: A Single-center Experience With 483 Patients. Annals of surgery 2017, 265(6):1219-1225.

25. Konstantinidis IT, Warshaw AL, Allen JN, Blaszkowsky LS, Castillo CF, Deshpande V, Hong TS, Kwak EL, Lauwers GY, Ryan DP et al: Pancreatic ductal adenocarcinoma: is there a survival difference for R1 resections versus locally advanced unresectable tumors? What is a "true" R0 resection? Annals of surgery 2013, 257(4):731-736.

26. Hank T, Sandini M, Ferrone CR, Rodrigues C, Weniger M, Qadan M, Warshaw AL, Lillemoe KD, Fernandez-Del Castillo C: Association Between Pancreatic Fistula and Long-term Survival in the Era of Neoadjuvant Chemotherapy. JAMA surgery 2019, 154:943-951.

27. Hank T, Sandini M, Ferrone CR, Rodrigues C, Weniger M, Qadan M, Warshaw AL, Lillemoe KD, Fernandez-Del Castillo C: Association Between Pancreatic Fistula and Long-term Survival in the Era of Neoadjuvant Chemotherapy. JAMA surgery 2019.

28. Delpero JR, Bachellier P, Regenet N, Le Treut YP, Paye F, Carrere N, Sauvanet A, Autret A, Turrini O, MongesRanchin G et al: Pancreaticoduodenectomy for pancreatic ductal adenocarcinoma: a French multicentre prospective evaluation of resection margins in 150 evaluable specimens. HPB (Oxford) 2014, 16(1):20-33.

29. Gebauer F, Tachezy M, Vashist YK, Marx AH, Yekebas E, Izbicki JR, Bockhorn M: Resection margin clearance in pancreatic cancer after implementation of the Leeds Pathology Protocol (LEEPP): clinically relevant or just academic? World J Surg 2015, 39(2):493-499.

30. Delpero JR, Jeune F, Bachellier P, Regenet N, Le Treut YP, Paye F, Carrere N, Sauvanet A, Adham M, Autret A et al: Prognostic Value of Resection Margin Involvement After Pancreaticoduodenectomy for Ductal Adenocarcinoma: Updates From a French Prospective Multicenter Study. Annals of surgery 2017, 266(5):787796.

31. Hirono S, Kawai M, Okada K, Miyazawa M, Shimizu A, Kitahata Y, Ueno M, Yamaue H: Pancreatic neck cancer has specific and oncologic characteristics regarding portal vein invasion and lymph node metastasis. Surgery 2016, 159(2):426-440.

32. O'Sullivan AW, Heaton N, Rela M: Cancer of the uncinate process of the pancreas: surgical anatomy and clinicopathological features. Hepatobiliary \& pancreatic diseases international : HBPD INT 2009, 8(6):569-574. 
33. Tan X, Sivakumar S, Bednarsch J, Wiltberger G, Kather JN, Niehues J, de Vos-Geelen J, Valkenburg-van lersel L, Kintsler S, Roeth A et al: Nerve fibers in the tumor microenvironment in neurotropic cancer-pancreatic cancer and cholangiocarcinoma. Oncogene 2021, 40(5):899-908.

34. Meng Z, Cao M, Zhang Y, Liu Z, Wu S, Wu H: Tumor location as an indicator of survival in T1 resectable pancreatic ductal adenocarcinoma: a propensity score-matched analysis. BMC Gastroentero/ 2019, 19(1):59.

\section{Tables}

Table 1 Distribution of the $\mathrm{R}$ status in patients who underwent PD and DP 


\begin{tabular}{|c|c|c|c|c|c|c|c|c|}
\hline \multirow[t]{2}{*}{ (\%) } & \multicolumn{3}{|c|}{ R status of PD } & \multirow[t]{2}{*}{ Total } & \multicolumn{3}{|c|}{ R status of DP } & \multirow[t]{2}{*}{ Total } \\
\hline & Ro & $\mathrm{R} 1<1 \mathrm{~mm}$ & $\begin{array}{l}\text { R1- } \\
\text { Directed }\end{array}$ & & RO & $\mathrm{R} 1<1 \mathrm{~mm}$ & $\begin{array}{l}\text { R1- } \\
\text { Directed }\end{array}$ & \\
\hline & 166(69.2) & $42(17.5)$ & $32(13.3)$ & $240(100)$ & $94(64.4)$ & $35(24.0)$ & 17(11.6) & $146(100)$ \\
\hline \multicolumn{9}{|l|}{$\begin{array}{l}\text { Number of } \\
\text { R1 Margin }\end{array}$} \\
\hline 0 & 166(100) & $0(0)$ & $0(0)$ & $166(69.2)$ & $94(100)$ & $0(0)$ & $0(0)$ & $94(64.4)$ \\
\hline 1 & $0(0)$ & $33(78.6)$ & 19(59.4) & $52(21.7)$ & $0(0)$ & 23(65.7) & 12(70.6) & $35(24.0)$ \\
\hline 2 & $0(0)$ & $9(21.4)$ & $5(15.6)$ & 14(5.8) & $0(0)$ & 12(34.3) & $5(29.4)$ & 17(11.6) \\
\hline$\geq 3$ & $0(0)$ & $0(0)$ & $8(25.0)$ & $8(3.3)$ & & & & \\
\hline \multicolumn{9}{|l|}{$\begin{array}{l}\text { Transection } \\
\text { margin }\end{array}$} \\
\hline RO & 166(100) & $38(90.5)$ & $27(84.4)$ & $231(96.3)$ & $94(100)$ & $34(97.1)$ & 15(88.2) & $143(97.9)$ \\
\hline $\mathrm{R} 1<1 \mathrm{~mm}$ & $0(0)$ & $4(9.5)$ & $0(0)$ & $4(1.7)$ & $0(0)$ & $1(2.9)$ & $0(0)$ & $1(0.7)$ \\
\hline R1-Directed & $0(0)$ & $0(0)$ & $5(15.6)$ & $5(2.1)$ & $0(0)$ & $0(0)$ & $2(11.8)$ & $2(1.4)$ \\
\hline \multicolumn{9}{|l|}{$\begin{array}{l}\text { Anterior } \\
\text { surface }\end{array}$} \\
\hline RO & $166(100)$ & $29(69.0)$ & $20(62.5)$ & 215(89.6) & $94(100)$ & $8(22.9)$ & $6(35.3)$ & 108(74) \\
\hline $\mathrm{R} 1<1 \mathrm{~mm}$ & $0(0)$ & 13(31.0) & $2(6.3)$ & $15(6.3)$ & $0(0)$ & $25(71.4)$ & $0(0)$ & $25(17.1)$ \\
\hline R1-Directed & $0(0)$ & $0(0)$ & 10(31.3) & $10(4.2)$ & $0(0)$ & $2(5.7)$ & 11(64.7) & $13(8.9)$ \\
\hline \multicolumn{9}{|l|}{$\begin{array}{l}\text { Posterior } \\
\text { margin }\end{array}$} \\
\hline RO & $166(100)$ & $32(76.2)$ & 19(59.4) & $217(90.4)$ & $94(100)$ & $16(45.7)$ & $8(47.1)$ & 118(80.8) \\
\hline $\mathrm{R} 1<1 \mathrm{~mm}$ & $0(0)$ & $10(23.8)$ & $5(15.6)$ & $15(6.3)$ & $0(0)$ & 19(54.3) & $0(0)$ & 19(13.0) \\
\hline R1-Directed & $0(0)$ & $0(0)$ & $8(25.0)$ & $8(3.3)$ & $0(0)$ & $0(0)$ & $9(52.9)$ & $9(6.2)$ \\
\hline \multicolumn{9}{|l|}{$\begin{array}{l}\text { Medial } \\
\text { margin }\end{array}$} \\
\hline RO & $166(100)$ & $18(42.9)$ & $9(28.1)$ & 193(80.4) & & & & \\
\hline $\mathrm{R} 1<1 \mathrm{~mm}$ & $0(0)$ & $24(57.1)$ & $5(15.6)$ & $29(12.1)$ & & & & \\
\hline R1-Directed & $0(0)$ & $0(0)$ & $18(56.3)$ & $18(7.5)$ & & & & \\
\hline \multicolumn{9}{|l|}{$\begin{array}{l}\text { Bile duct } \\
\text { margin }\end{array}$} \\
\hline R0 & 166(100) & $42(100)$ & $30(93.8)$ & 238(99.2) & & & & \\
\hline $\mathrm{R} 1<1 \mathrm{~mm}$ & $0(0)$ & $0(0)$ & 1(3.1) & $1(0.4)$ & & & & \\
\hline R1-Directed & $0(0)$ & $0(0)$ & 1(3.1) & $1(0.4)$ & & & & \\
\hline
\end{tabular}


margins

R0 166(100) 42(100) 32(100) 240(100)

Table 2 Univariate analysis of risk factors for $\mathrm{R} 1$ resection 


\begin{tabular}{|c|c|c|c|c|c|c|}
\hline & PD & & & DP & & \\
\hline & R status & & $P$ & R status & & $P$ \\
\hline & Ro & R1 & & Ro & R1 & \\
\hline Total $(\%)$ & $166(69.2)$ & $74(30.8)$ & & $94(64.4)$ & $52(35.6)$ & \\
\hline Gender & & & 0.237 & & & 0.489 \\
\hline Male(\%) & $101(60.8)$ & $39(52.7)$ & & $56(59.6)$ & $34(65.4)$ & \\
\hline Female(\%) & $65(39.2)$ & $35(47.3)$ & & $38(40.4)$ & 18(34.6) & \\
\hline Age & & & 0.896 & & & 0.799 \\
\hline$\llbracket 60$ years(\%) & $53(31.9)$ & 23(31.1) & & $40(42.6)$ & $21(40.4)$ & \\
\hline$\geq 60$ years $(\%)$ & 113(68.1) & $51(68.9)$ & & $54(57.4)$ & $31(59.6)$ & \\
\hline Preoperative CA19-9 & & & 0.415 & & & 0.351 \\
\hline$\varangle 223 \mathrm{U} / \mathrm{mL}(\%)$ & $110(66.3)$ & $45(60.8)$ & & $65(69.1)$ & $32(61.5)$ & \\
\hline$\geq 223 \mathrm{U} / \mathrm{mL}(\%)$ & $56(33.7)$ & $29(39.2)$ & & $29(30.9)$ & $20(38.5)$ & \\
\hline Location & & & $<0.001$ & & & 0.214 \\
\hline Head(\%) & 139(83.7) & $44(59.5)$ & & & & \\
\hline $\operatorname{Neck}(\%)$ & $3(1.8)$ & 10(13.5) & & & & \\
\hline Uncinate (\%) & $24(14.5)$ & $20(27.0)$ & & & & \\
\hline Neck and body (\%) & & & & $11(11.7)$ & $10(19.2)$ & \\
\hline Body and tail (\%) & & & & $83(88.3)$ & $42(80.8)$ & \\
\hline Resectability status & & & 0.529 & & & 0.996 \\
\hline Resectable(\%) & 131(78.9) & $61(82.4)$ & & $56(59.6)$ & $31(59.6)$ & \\
\hline Borderline resectable(\%) & $35(21.1)$ & 13(17.6) & & $38(40.4)$ & $21(40.4)$ & \\
\hline Vascular resection & & & 0.934 & & & 0.211 \\
\hline Without(\%) & 142(85.5) & 63(85.1) & & $77(81.9)$ & $38(73.1)$ & \\
\hline With (\%) & $24(14.5)$ & $11(14.9)$ & & $17(18.1)$ & $14(26.9)$ & \\
\hline Necrosis & & & 0.050 & & & 0.466 \\
\hline Without(\%) & 18(10.8) & $15(20.3)$ & & $15(16.0)$ & $6(11.5)$ & \\
\hline With (\%) & $148(89.2)$ & $59(79.7)$ & & $79(84.0)$ & $46(88.5)$ & \\
\hline Perineural invasion & & & 0.100 & & & 0.041 \\
\hline Without(\%) & $14(8.4)$ & $2(2.7)$ & & $14(14.9)$ & $2(3.8)$ & \\
\hline With (\%) & 152(91.6) & 71(97.3) & & $80(85.1)$ & $50(96.2)$ & \\
\hline Lymphovascular invasion & & & 0.054 & & & 0.617 \\
\hline
\end{tabular}




\begin{tabular}{|c|c|c|c|c|c|c|}
\hline Without(\%) & $105(63.3)$ & $37(50)$ & & $67(71.3)$ & $35(67.3)$ & \\
\hline With (\%) & $61(36.7)$ & $37(50)$ & & $27(28.7)$ & $17(32.7)$ & \\
\hline Duodenal papilla invasion & & & 0.076 & & & \\
\hline Without(\%) & 119(71.7) & $61(82.4)$ & & & & \\
\hline With (\%) & $47(28.3)$ & $13(17.6)$ & & & & \\
\hline G & & & 0.374 & & & 0.714 \\
\hline $\mathrm{G} 1 / 2(\%)$ & 134(80.7) & $56(75.7)$ & & $66(70.2)$ & $38(73.1)$ & \\
\hline $\mathrm{G} 3 / 4(\%)$ & $32(19.3)$ & $18(24.3)$ & & $28(29.8)$ & $14(26.9)$ & \\
\hline T stage & & & 0.404 & & & $<0.001$ \\
\hline $1 / 2(\%)$ & 134(80.7) & $54(73.0)$ & & $54(57.4)$ & $12(23.1)$ & \\
\hline $3(\%)$ & $32(19.3)$ & $20(27.0)$ & & $40(42.6)$ & $40(76.9)$ & \\
\hline $\mathrm{N}$ stage & & & $<0.001$ & & & 0.095 \\
\hline $0(\%)$ & $71(42.8)$ & $12(16.2)$ & & $41(43.6)$ & $16(30.8)$ & \\
\hline $1(\%)$ & $60(36.1)$ & $27(36.5)$ & & $39(41.5)$ & $21(40.4)$ & \\
\hline $2(\%)$ & $35(21.1)$ & $35(47.3)$ & & $14(14.9)$ & $15(28.8)$ & \\
\hline TNM stage & & & $<0.001$ & & & 0.038 \\
\hline \/凶(\%) & $130(78.3)$ & $38(51.4)$ & & $76(80.9)$ & $34(65.4)$ & \\
\hline$\bigotimes(\%)$ & $36(21.7)$ & $36(48.6)$ & & $18(19.1)$ & $18(34.6)$ & \\
\hline
\end{tabular}

Table 3 Multivariate analysis of risk factors for R1 resection

\begin{tabular}{|lllll|}
\hline \multicolumn{4}{|c|}{$\mathrm{PD}$} & \multicolumn{2}{c|}{$\mathrm{DP}$} \\
\hline & $\mathrm{OR}(95 \% \mathrm{Cl})$ & $P$ & $\mathrm{OR}(95 \% \mathrm{Cl})$ & $P$ \\
\hline T stage & & & & \\
\hline T1/2 v.s. T3 & & & $5.26(2.36-11.73)$ & $<0.001$ \\
\hline N stage & & & \\
\hline N1 v.s. N0 & $2.41(1.08-5.35)$ & $\mathbf{0 . 0 3 1}$ & \\
\hline N2 v.s. N0 & $6.43(2.85-14.50)$ & $<0.001$ & \\
\hline Location & & & \\
\hline Neck v.s. head & $9.55(2.39-38.20)$ & $\mathbf{0 . 0 0 1}$ & \\
\hline Uncinate v.s. head & $3.31(1.58-6.94)$ & $\mathbf{0 . 0 0 2}$ & \\
\hline
\end{tabular}



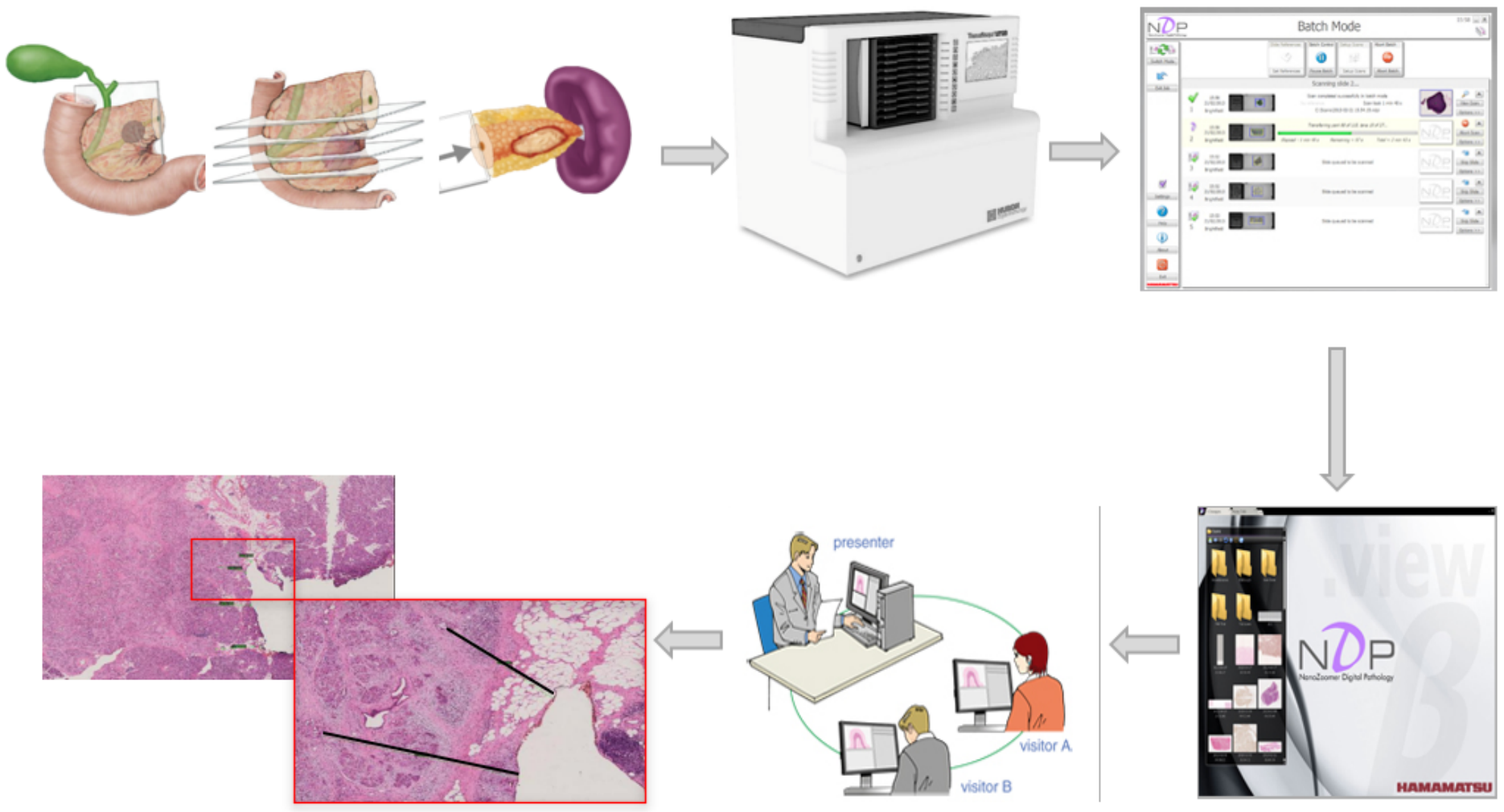

\section{Figure 1}

Schematic representation of a standardized pathological examination for the $\mathrm{R}$ status which includes: the entire specimen being sliced and made into whole-tissue glass slides, the whole-tissue glass slides area then scanned by the Hamamatsu S60 whole slide scanner. The software NDP viewer is then used to obtain the DWMSIs, which is viewed by the NDP viewer. The pathologists view the DWMSIs on the screen, and measure the distance between the margins and the tumor cells to identify the R status. NDP: NanoZoomer Digital Pathology, DWMSIs: digital wholemount slide images. 


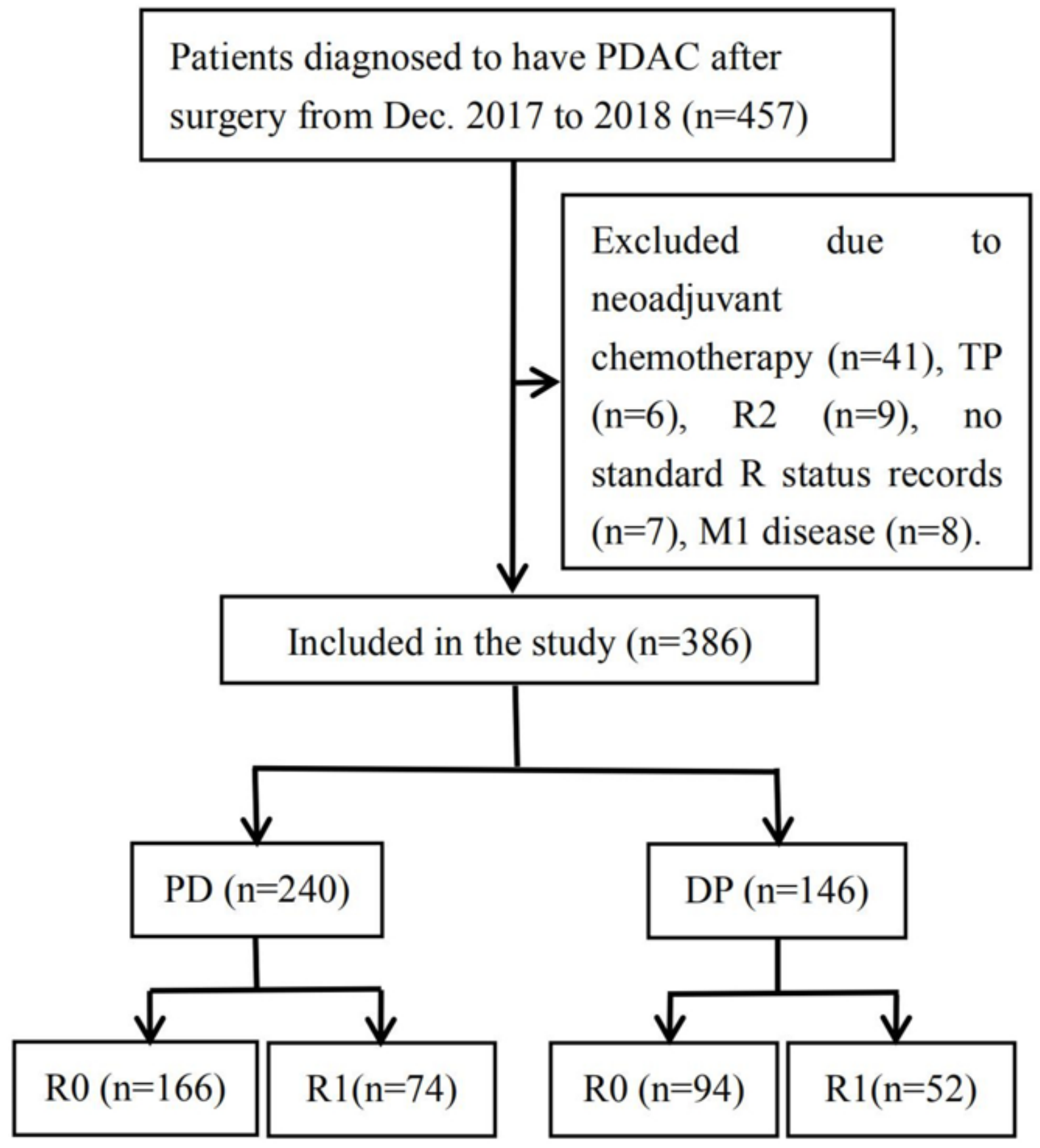

Figure 2

Flowchart of patient selection in the study. DP: distal pancreatosplenectomy, PD: pancreaticoduodenectomy, TP: total pancreatectomy. 

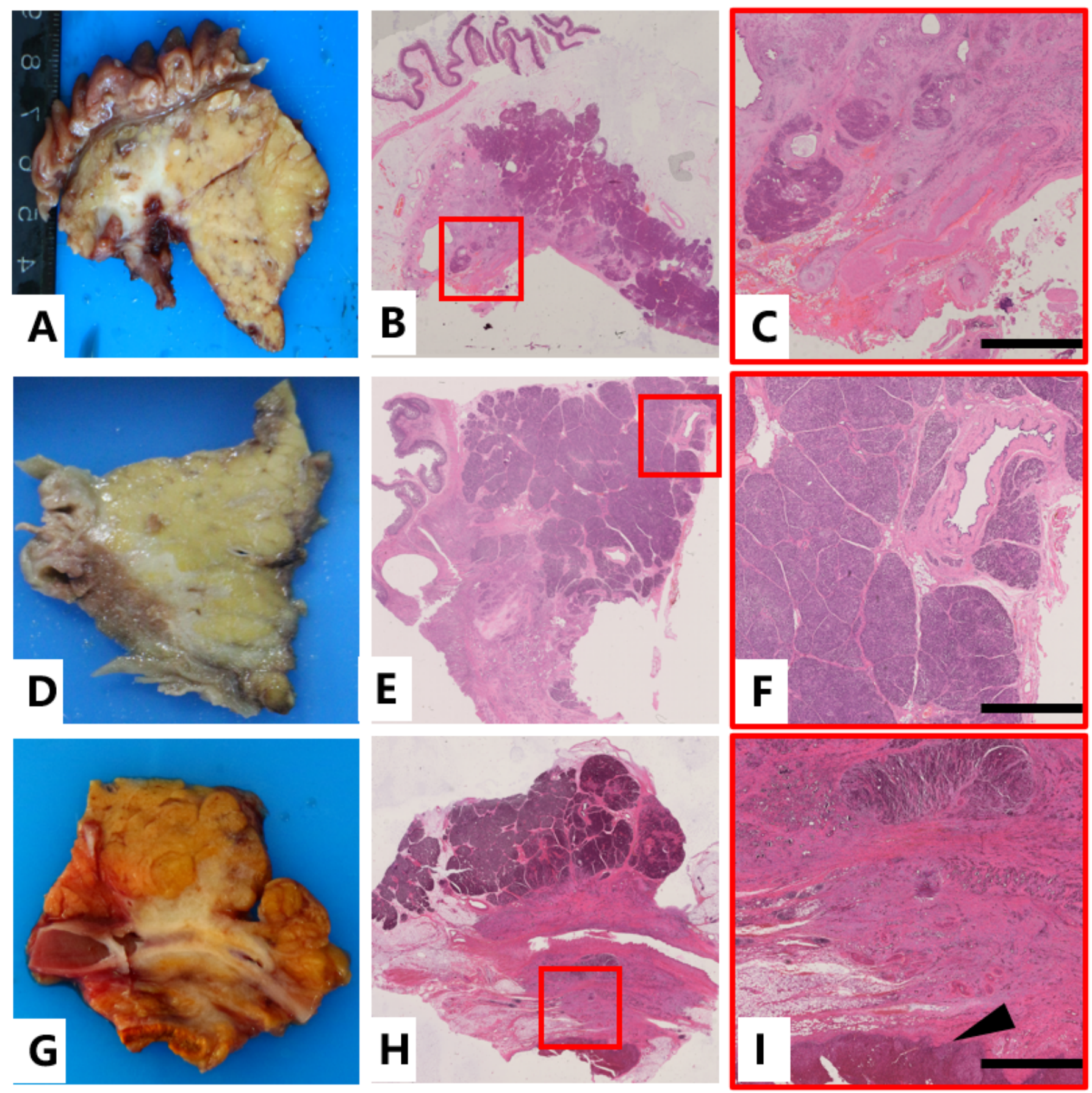

\section{Figure 3}

$\mathrm{R}$ status identification for specific cases. A-C show a tumor located in the uncinate process and the medial margin was identified as R1-directed. D-F show a tumor located in the head and the posterior margin was identified as R0. $\mathrm{H}-\mathrm{I}$ show a specimen obtained from posterior RAMPS (black arrow shows the adrenal gland in the specimen in $\mathrm{H}$ ) and the tumor cells had infiltrated into the left adrenal gland (black arrowhead). Slides B,C,E,F,H and I are stained with hematoxylin-eosin. Scale marker, $3 \mathrm{~mm}$. RAMPS: radical antegrade modular pancreatosplenectomy.

\section{Supplementary Files}

This is a list of supplementary files associated with this preprint. Click to download. 
- SupplementaryFigure1.png

- SupplementaryTables.docx 